\title{
Préparations galactogènes utilisées par les agroéleveurs au Bénin : espèces végétales, proportions d'organes impliqués et production laitière chez les vaches Borgou
}

\author{
AGANI Zénabou ${ }^{1 *}$, BOKO K. Cyrille 3 , AKOUEDEGNI C. Guénole², SIDI IMOROU Habirou ${ }^{1}$, BELLO Orou \\ Daouda ${ }^{5}$, HOUNDONOUGBO M. Frédéric ${ }^{6}$, DOSSOU Joseph ${ }^{4}$, BABATOUNDE Séverin ${ }^{1}$ \\ ${ }^{1}$ Laboratoire de Zootechnie, Faculté des Sciences Agronomiques, Université d'Abomey-Calavi, 01 BP 526, Cotonou, \\ 2Laboratoire d'Ethnopharmacologie et de Santé Animale, FSA/UAC \\ 3Unité de Recherche sur les Maladies Transmissibles, EPAC/UAC \\ 4Laboratoire de Bio ingénierie des Procédés Alimentaires LaBioPA/FSA/UAC \\ 5 Laboratoire de Biologie Végétale (LBV)/FSA \\ ${ }^{6}$ Laboratoire de Recherche Aviaire et de Zoo-économie LaRAZE/FSA \\ E-mail de l'auteur correspondant : zenabagani@gmail.com
}

Original submitted in on $2^{\text {nd }}$ November 2020. Published online at www.m.elewa.org/journals/ on 31st January 2021 https://doi.org/10.35759/JABs.157.2

\begin{abstract}
RÉSUMÉ
Objectif : Au Bénin, la production de lait des vaches locales ne permet pas de couvrir les besoins nutritionnels de la population. Pour l'améliorer, les agroéleveurs font recours à des recettes galactogènes. Cette étude évalue leur efficacité sur la production laitière des vaches de race Borgou.

Méthodologie et Résultats : Au Bénin, une enquête réalisée auprès de 534 éleveurs a permis de recenser 286 recettes galactogènes utilisées par les agroéleveurs. Sur la base d'une classification numérique des recettes citées par au moins $20 \%$ des enquêtés, dix (10) recettes ont été retenues pour des enquêtes approfondies. Bobgunnia madagascariensis, Euphorbia balsamifera, Curcubita maxima sont fréquemment impliquées dans les préparations galactogènes. Les racines et les graines sont les organes les plus utilisés. Les graines de Vigna unguiculata ou d'Arachis Hypogea sont toujours associées aux préparations. La production laitière est plus élevée après administration aux vaches et peut atteindre 2,5 litres par jour.

Conclusion et application des résultats : L'étude confirme l'efficacité des préparations galactogènes utilisées par les agroéleveurs pour améliorer la production laitière des vaches locales. II est intéressant de constater que les espèces végétales (Bobgunnia madagascariensis, Curcubita maxima, Euphorbia basalmifera) fréquemment utilisées sont présentes dans la flore béninoise. Les graines de Vigna unguiculata ou d'Arachis Hypogea sont toujours associées aux préparations galactogènes. Les racines et les graines sont les organes les plus utilisés. Quelle que soit la recette utilisée, la production du lait est plus élevée après administration des préparations aux vaches $(P<0,05)$ et peut toutefois atteindre 2,5 litres contre 1,5 par jour relevée en élevage semi-intensif pour les races locales. Le gain laitier suite à l'administration des recettes s'étale entre 0,6 et 1,1 litre par jour. II ne reste maintenant qu'à identifier pour les recettes la forme galénique la mieux adaptée aux conditions socio-économiques de ces éleveurs traditionnels.

Mots-clés : Bobgunnia madagascariensis, Euphorbia balsamifera, Curcubita maxima, recette galactogènes, vaches Borgou, Bénin.
\end{abstract}


Agani et al., J. Appl. Biosci. 2021 Préparations galactogènes utilisées par les agroéleveurs au Bénin : espèces végétales, proportions d'organes impliqués et production laitière chez les vaches Borgou

Galactogenic formulations used by agro-pastoralists in Benin: plant species, proportions of organs involved, and milk production in Borgou cows.

\begin{abstract}
Objective: In Benin, the milk production of local cows does not cover the nutritional needs of the population. To improve it, agro-pastoralists use galactogenic formulations. This study evaluates their effectiveness on the milk production of Borgou cows.

Methodology and Results: In Benin, a survey of 534 breeders identified 286 galactogenic formulations used by agro-breeders. Based on a numerical classification of the formulations cited by at least $20 \%$ of respondents, ten (10) formulations were retained for in-depth investigations. Bobgunnia madagascariensis, Euphorbia balsamifera, Curcubita maxima were frequently involved in these galactogenic preparations. The roots and seeds are the most used organs. The seeds of Vigna unguiculata or Arachis Hypogea were also always associated with preparations. Milk production was higher after administration to cows and could reach 2.5 litres per day.

Conclusion and application of the results: The study confirms the effectiveness of galactogenic feed formulations used by agro-breeders to improve milk production in local cows. It is interesting to note that the plant species (Bobgunnia madagascariensis, Curcubita maxima, Euphorbia basalmifera) frequently used are present in the Beninese flora. The seeds of Vigna unguiculata or Arachis Hypogea are always associated with galactogenic preparations. The roots and seeds are the most used organs. Whatever the recipe used, the milk production is higher after administration of the formulas to the cows $(P<0.05)$ and can however reach 2.5 litres against 1.5 per day observed in semi-intensive breeding for local breeds.. The milk gain from the administration of the recipes ranges from 0.6 to 1.1 litres per day. All that remains now is to identify the dosage form best suited to the socio-economic conditions of these traditional breeders for the recipes.

Keywords: Bobgunnia madagascariensis, Euphorbia balsamifera, Curcubita maxima, galactogenic recipe, Borgou cows, Benin.
\end{abstract}

\title{
INTRODUCTION
}

Au Bénin, les productions animales restent encore marquées par les pratiques traditionnelles d'élevage des espèces bovines, ovines, caprines, porcines et des volailles, malgré les résultats assez concluants des projets d'élevage moderne au cours de la dernière décennie. Dans ce pays, l'élevage bovin revêt une grande importance pour les populations du nord Bénin qui en font leur principale activité. Traditionnellement, ce sont les peuhls qui s'occupent de l'élevage. Ils sont pasteurs et possèdent plus de $85 \%$ du cheptel national bovin estimé à 2166000 têtes (FAO, 2013). De l'exploitation de ce cheptel provient le lait de vache qui est le plus utilisé dans les habitudes alimentaires. En milieu d'élevage traditionnel, le lait appartient à la femme et constitue sa principale source de revenu. Les recettes issues de la vente du lait et des produits dérivés sont utilisées pour l'approvisionnement en condiments pour l'alimentation du ménage. Le reste des recettes est utilisé pour les besoins domestiques de la femme et des enfants. Le chef de famille n'a pas accès direct à ce revenu (Mama Sambo, 2013). Ces dispositions montrent le rôle que joue le lait dans la sécurité alimentaire des ménages traditionnels et sur le plan social. Toutefois, la production locale à elle seule n'est pas suffisante au regard des besoins de la population. Le lait est consommé sous toutes ses formes. On retrouve du yaourt (nature, fruité et associé au mil communément appelé " dèguê », le lait cru (entier, demi écrémé, écrémé ou caillé), du beurre, du fromage localement, un fromage frais moulé appelé «Wagaashi ». Les vaches de race locale ne produisent que 210 litres pour une durée moyenne de lactation de 180 jours. Au regard de cette faible performance des vaches locales, de l'importance nutritionnelle, économique et sociale du lait dans les milieux d'élevage traditionnel, les éleveurs se réfèrent aux connaissances endogènes, notamment l'utilisation des 
préparations galactogènes pour améliorer sa production. De nombreuses recherches ont été réalisées sur les connaissances ethno-vétérinaires des éleveurs. Elles confirment l'utilisation des pratiques endogènes par ceux-ci pour induire ou améliorer la production chez les mammifères y compris l'homme (Akouedegni et al., 2012 ; Dassou et al., 2014 ; Dossou et al., 2012; Koko et al., 2011). Récemment, (Salifou et al., 2017) ont mené des enquêtes sur les plantes lactogènes des bovins et

\section{MATÉRIEL ET MÉTHODES}

L'étude a été menée au Bénin dans les communes de Gogounou, de Kalalé et de Djidja. La forte concentration des bovins, l'existence de grands marchés à bétail et d'une variété d'espèces végétales entrant dans la préparation des recettes galactogènes sont les raisons qui ont milité en faveur du choix de ces communes. La commune de Kalalé occupe est située entre $3^{\circ} 22^{\prime} 54^{\prime \prime}$ de longitude Est et $10^{\circ} 17^{\prime} 24^{\prime \prime}$ de latitude Nord. Le climat est de type soudanien, avec une alternance de pluie (avril à octobre) et de saison sèche (Novembre à Mars). La pluviométrie annuelle varie entre 845 et $1322 \mathrm{~mm}$, et suit une distribution unimodale. Le système d'utilisation de la terre est basé sur le sorgho et l'igname, avec une forte concentration en maïs et en coton. La commune de Gogounou, est comprise entre les parallèles $10^{\circ} 29^{\prime} 34^{\prime \prime}$ et $11^{\circ} 45^{\prime} 25^{\prime \prime}$ de latitude Nord et les méridiens $01^{\circ} 42^{\prime} 54^{\prime \prime}$ et $3^{\circ} 43^{\prime} 51^{\prime \prime}$ de longitude Est (Figure 1). Encore appelé le grenier du Zou, la commune de Djidja est comprise entre les parallèles $7^{\circ} 10^{\prime}$ et $7^{\circ} 40^{\prime}$ de latitude Nord, et les méridiens $1^{\circ} 04^{\prime}$ et $2^{\circ} 10^{\prime}$ de longitude Est et caractérisée par un climat de type subéquatorial tendant vers le soudano-guinéen dans les parties septentrionales avec deux saisons pluvieuses. L'enquête a été réalisée en pleine saison pluvieuse (avril à septembre 2019), auprès de 534 au moyen d'interviews directes. Sur les 286 recettes galactogènes recensées, une classification numérique a été faite sur celles ayant été citées par au moins $20 \%$ des enquêtés. Sur cette base, dix (10) recettes ont été retenues pour des enquêtes beaucoup plus approfondies allant de novembre 2019 à février 2020. Les éleveurs ont été suivis en milieu réel et les productions de lait ont été quantifiées avant et après l'administration des recettes sur des vaches Borgou élevés en milieu réel. Le matériel végétal était constitué de plantes collectées dans les forêts environnantes des zones d'étude. Ces plantes ont été collectées tôt le matin ou avant le coucher du soleil. L'identification taxonomique a été réalisée à l'herbier national du Bénin leurs modes de préparation dans les élevages traditionnels. Ces travaux ne précisent pas les proportions des organes impliqués, les doses administrées ainsi que les quantités de lait obtenues suite à l'administration des préparations aux vaches. Pour ce faire, l'objectif général du travail est de faire une étude diagnostique sur des préparations galactogènes utilisées par les éleveurs traditionnels pour améliorer la production du lait des vaches locales élevées en milieu.

par comparaison avec les herbiers de référence avec la flore analytique du Bénin (Akouègninou et al., 2006). Le matériel animal était composé de 10 lots de 6 vaches dont 3 témoins et 3 vaches expérimentales de poids moyen $200 \mathrm{~kg}$ et de rang de vêlage 2. Par ailleurs, les sujets ont reçu $48 \mathrm{~h}$ avant l'expérimentation un traitement général à l'aide d'oxytrétracycline $20 \%$ pour éviter les surinfections éventuelles, un lavage sanguin à base de Vériben B12, de la vitamine contre le stress et un déparasitant interne et externe à base d'Ivomec. Les sujets étaient nourris avec des résidus de récolte complémentés par un apport minéral (sel et de pierre à lécher). Pour décrire le mode de préparation des recettes utilisées par chaque éleveur, les quantités des ingrédients utilisés ont été mesurées à l'aide d'une balance de portée $10 \mathrm{~kg} \pm 5 \mathrm{~g}$. Une éprouvette de $2 \mathrm{~L} \mathrm{a}$ été également utilisée pour mesurer les diluants. Les données collectées étaient: l'âge des veaux, le périmètre mammaire avant et après administration des préparations galactogènes, la quantité de lait produite pendant 3 jours avant l'administration et 7 jours après l'administration des préparations galactogènes.

Analyse statistique: La Cpr est la contribution de chaque plante dans la constitution des recettes. C'est aussi la Fréquence d'implication des plantes dans les recettes. Elle a été calculée par la formule :

$\mathrm{Cpr}=\frac{N c r}{N t}$ où Ncr est le nombre de recettes sollicitant la plante et Nt le nombre total de recettes.

Ensuite, les données collectées relatives à l'étude diagnostique ont été codifiées, saisies et traitées avec logiciel $\mathrm{R}$ version 2.03. La statistique descriptive en termes de pourcentage, de moyenne, d'écart-type et de coefficient de variation a été utilisée pour les données relatives à la recette, la production laitière et les poids des animaux. Par la suite, ces paramètres ont été soumis à une analyse de la variance à deux critères de classification (la recette, production laitière de l'animal) 
en utilisant la procédure PROC GLM du logiciel SAS (Statistical Analysis System) version 9.2 (SAS inc., NC, USA). Au terme de l'analyse, les différences entre les

\section{RÉSULTATS}

Espèces végétales, organes utilisés et mode de préparation des recettes galactogènes : Au tableau 1 sont présentés, les différents organes utilisés pour chaque espèce impliquée dans la recette, les quantités des organes, les doses administrées ainsi que les durées d'utilisation. Quelle que soit la partie de la plante impliquée dans la recette, trois espèces végétales (Bobgunnia madagascariensis, Euphorbia balsamifera, Curcubita maxima) ont été fréquemment impliquées dans les préparations des recettes et elles intervenaient en proportion beaucoup plus élevée que les autres espèces. Bobgunnia madagascariensis et Euphorbia balsamifera étaient les espèces végétales les plus utilisées dans la préparation des recettes galactogènes. Une légumineuse à graine, notamment Vigna unguiculata étaient toujours associée à la préparation. Le mode de préparation de ces recettes était essentiellement la décoction $(60 \%)$ et la macération $(40 \%)$. En ce qui concerne la décoction, les ingrédients végétaux ont été lavés après cueillette dans une grande marmite selon la quantité à administrer et le nombre de jours de traitement des animaux. Ces végétaux auxquels les éleveurs ajoutaient des graines pilées de Vigna unguiculata et/ou Arachis hypogea ont été portés à ébullition pendant $30 \mathrm{mn}$. Ils étaient ensuite baratés pendant $10 \mathrm{mn}$. La quantité des préparations à administrer à l'animal ne dépassait pas 1 litre. Cette quantité a été mesurée à l'aide des plastiques de boisson recyclés ou des calebasses qui servent à traire le lait. L'administration de la recette à l'animal se faisait par gavage d'une dose qui variait entre 0,5 et 1 litre. Quant au mode de préparation par macération, les ingrédients ont été pilés dans un mortier artisanal puis moyennes sont comparées selon le test de Newman et Keuls (Dagnelie, 1973).

baratés pendant $15 \mathrm{mn}$. Les quantités des ingrédients prélevés en vue de la préparation des recettes galactogènes étaient mesurées à l'aide du poignet ou des calebasses servant à traire. Généralement, les quantités administrées aux animaux variaient d'un élevage à un autre. Elles dépendaient essentiellement de l'expertise de l'éleveur et de certains paramètres zootechniques notamment, le niveau de production, l'âge, l'état corporel de l'animal. Quoi qu'il en soit, les durées d'utilisation des recettes n'excédaient pas 7 jours. Comme on peut le remarquer à travers les résultats du tableau1 et ceux rendus aussi disponibles dans la figure 1 , les racines et les graines $(32,3 \%)$ étaient les organes les plus sollicités dans les préparations galactogènes. A titre d'illustration, les recettes R1 et R3 nécessitent l'implication de 46 et $67 \%$ respectivement de racines Bobgunnia madagascariensis et de graines de Vigna unguiculata. Dans les recettes où les feuilles interviennent, ce sont les jeunes feuilles des plantes qui étaient utilisées. Celles du Vitellaria paradoxa ont été fréquemment utilisées. Par contre, pour les recettes où les racines des plantes sont impliquées, l'espèce Bobgunnia madagascariensis (desv.) a été beaucoup plus sollicitée. Les éleveurs avaient indiqué que le moment de la cueillette des organes (matin ou au coucher du soleil) influençait l'efficacité de la recette. Par ailleurs, l'étude a montré que 12 espèces végétales appartenant à 8 différentes familles (tableau 2), dont les légumineuses (33\%), les apocyanacés et euphorbiacés (30\%).L'implication des autres familles (curcubitacés, poacés, sapotacés, rubiacés, moracés) dans les préparations galactogènes n'excédait pas $10 \%$. 
Agani et al., J. Appl. Biosci. 2021 Préparations galactogènes utilisées par les agroéleveurs au Bénin : espèces végétales, proportions d'organes impliqués et production laitière chez les vaches Borgou

Tableau 1. Espèces végétales, organes utilisés et quantités impliqués dans la constitution des recettes

\begin{tabular}{|c|c|c|c|c|c|c|c|}
\hline Recettes & Plantes & $\begin{array}{l}\text { Organes } \\
\text { utilisées }\end{array}$ & $\begin{array}{l}\text { Mode de } \\
\text { préparation }\end{array}$ & $\begin{array}{l}\text { Quantité d'organe } \\
\text { (g) }\end{array}$ & $\begin{array}{l}\text { Proportion } \\
\text { d'organe } \\
(\%)\end{array}$ & $\begin{array}{l}\text { Doses } \\
\text { administrées } \\
\text { (L) }\end{array}$ & $\begin{array}{l}\text { durée } \\
\text { d'utilisation }\end{array}$ \\
\hline R1 & $\begin{array}{l}\text { Bobgunnia madagascariensis + Vigna } \\
\text { unguiculata + Sorghum bicolor + Raphionacme } \\
\text { brownii+ Calotropis procera }\end{array}$ & $\begin{array}{l}\text { Racine, graine, } \\
\text { balle, tubercule, } \\
\text { racine }\end{array}$ & Décoction & $\begin{array}{l}(535+188+128+ \\
168+154)\end{array}$ & $\begin{array}{l}(46+16+11+ \\
14+13)\end{array}$ & 1 & 7 jours \\
\hline $\mathrm{R} 2$ & $\begin{array}{l}\text { Bobgunnia madagascariensis + Vigna } \\
\text { unguiculata }\end{array}$ & Racine, graine & Macération & $(84+168)$ & $(33+67)$ & 1 & 1 jour \\
\hline R3 & $\begin{array}{l}\text { Curcubita maxima + Euphorbia balsamifera + } \\
\text { Vitellaria paradoxa+Sorghum bicolor }\end{array}$ & $\begin{array}{l}\text { Fruit, plante, } \\
\text { entière, feuille } \\
\text { jeune, balle }\end{array}$ & Décoction & $\begin{array}{l}(897+308+136+ \\
210)\end{array}$ & $\begin{array}{l}(58+20+9+ \\
14)\end{array}$ & 1 & 3 jours \\
\hline R4 & Curcubita maxima+ Vigna unguiculata & Fruit, graine & Macération & $(1128+255)$ & $(82+18)$ & 0,5 & 3 jours \\
\hline R5 & $\begin{array}{l}\text { Bobgunnia madagascariensis + Arachis } \\
\text { hypogea + Vigna unguiculata }\end{array}$ & Racine, graine & Décoction & $(274+759+741)$ & $(15+43+42)$ & 0,5 & 2 jours \\
\hline $\mathrm{R} 6$ & $\begin{array}{l}\text { Gardenia aqualla + Euphorbia balsamifera + } \\
\text { Sorghum bicolor + Saba comorensis+ Curcubita } \\
\text { maxima+ Vigna unguiculata+ Arachis hypogea + } \\
\text { Calotropis procera + Vitellaria paradoxa }\end{array}$ & $\begin{array}{l}\text { Balle, racine, } \\
\text { fruit, graines } \\
\text { racine, jeune } \\
\text { feuille }\end{array}$ & Décoction & $\begin{array}{l}(189+185+153+ \\
252+791+178+ \\
221+266+200)\end{array}$ & $\begin{array}{l}(8+8+6+11 \\
+33+7+9+ \\
9+8)\end{array}$ & 1 & 5 jours \\
\hline $\mathrm{R} 7$ & $\begin{array}{l}\text { Bobgunnia madagascariensis + Vigna } \\
\text { unguiculata+ Sorghum bicolor }\end{array}$ & $\begin{array}{l}\text { Racine, graine, } \\
\text { son }\end{array}$ & Macération & $(369+624+635)$ & $(23+38+39)$ & 0,5 & 3 jours \\
\hline R8 & $\begin{array}{l}\text { Saba comorensis+ Curcubita maxima+ Ficus } \\
\text { trichopoda }\end{array}$ & $\begin{array}{l}\text { Racine, fruit, } \\
\text { racine }\end{array}$ & Décoction & $(335+913+253)$ & $(22+61+17)$ & 1 & 5 jours \\
\hline R9 & $\begin{array}{l}\text { Euphorbia basalmifera + Vigna unguiculata + } \\
\text { Arachis hypogea }\end{array}$ & $\begin{array}{l}\text { Tige, feuille, } \\
\text { graine }\end{array}$ & Macération & $(854+421+400)$ & $(51+25+24)$ & 1 & 5 jours \\
\hline R10 & Gardenia aqualla & Racine & Décoction & 895 & 100 & 0,5 & 3 jours \\
\hline
\end{tabular}

$\AA=$ Recette ; $(\mathrm{L})=$ Litre, la position de chaque organe et quantité d'ingrédient correspond au numéro d'ordre des espèces impliquées dans chaque recette 
Tableau 2 : Fréquence d'implication des espèces végétales entrant dans la constitution des préparations

\begin{tabular}{l|l|l|l}
\hline Noms scientifiques & Familles & Nom en peulhs & $\mathbf{F}_{\mathrm{cp}}$ \\
\hline Vigna unguiculata (I.) Walp & Leguminosae & $\underline{\text { Yièbè }}$ & 0,7 \\
Bobgunnia madagascariensis (desv.) & Leguminosae & Cocobit & 0,4 \\
Sorghum bicolor (I.) Moench & Poaceae & Dobi & 0,4 \\
Cucurbita maxima duchesne & Cucurbitaceae & Pampalocobial & 0,4 \\
Arachis hypogaea I. & Leguminosae & Biridji & 0,3 \\
Euphorbia balsamifera aiton & Euphorbiaceae & Tcholoui & 0,3 \\
Saba comorensis (bojer ex a.dc.) Pichon & Apocynaceae & Bernai & 0,2 \\
Calotropis procera subsp. Hamiltonii & Apocynaceae & Bambambi & 0,2 \\
Gardenia aqualla stapf \& hutch & Rubiaceae & Dihanli & 0,2 \\
Vitellaria paradoxa c.f.gaertn. Ssp. & Sapotaceae & Karé & 0,2 \\
Raphionacme brownii scott-elliot & Apocynaceae & Deyyol & 0,1 \\
Ficus trichopoda & Moraceae & llawadii & 0,1 \\
\hline
\end{tabular}

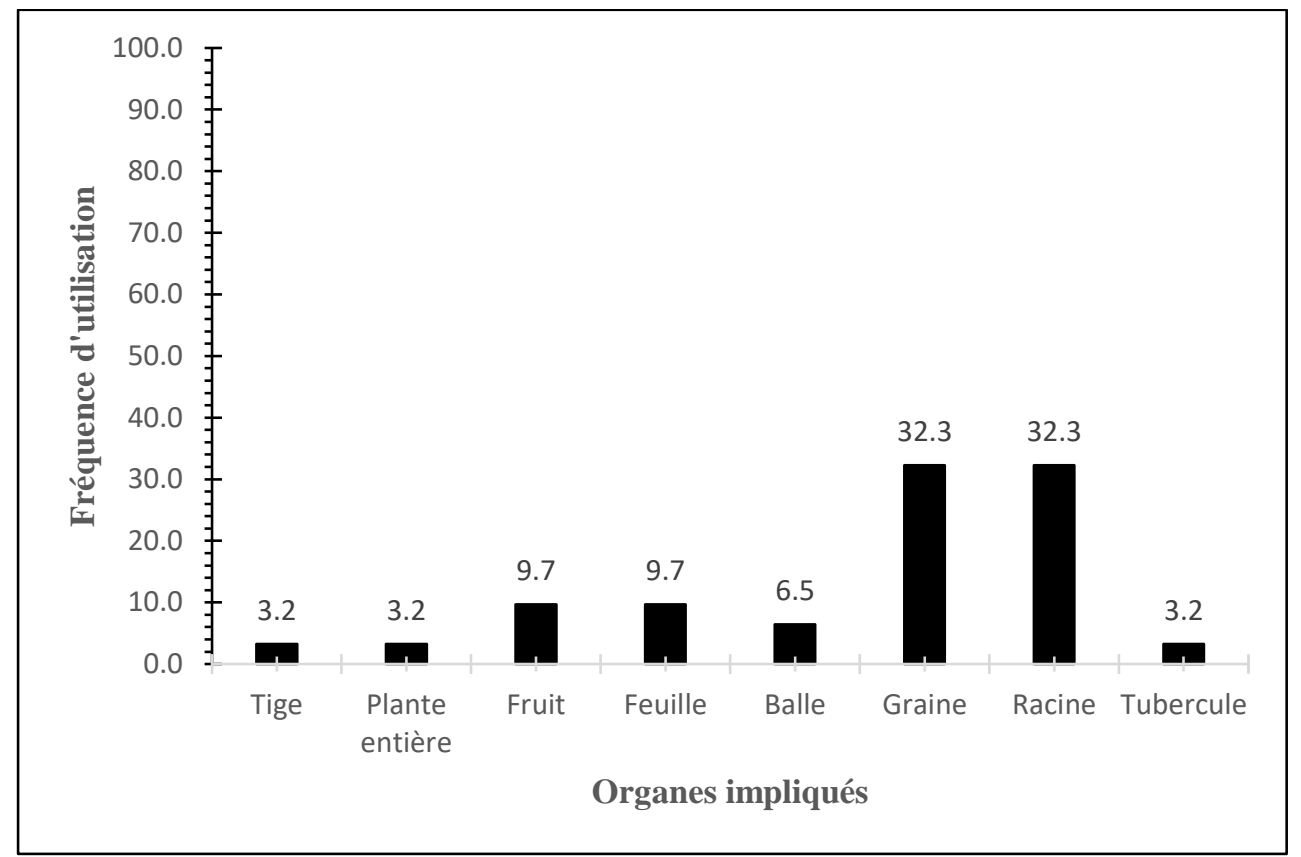

Figure 1 : Fréquence d'utilisation des organes de plantes impliquées dans les préparations galactogènes

Effet des recettes galactogènes sur la production laitière chez les vaches : Au Tableau 3 sont présentés les productions laitières avant et après administration de la recette ainsi que le gain laitier obtenu. Les valeurs de Fisher $(F)$ et les probabilités $(P)$ associées à chaque facteur sont aussi consignées dans le même tableau. Suite à l'administration de la recette aux vaches, la production du lait est plus élevée, quelle que soit la recette $(P<0,05)$. En moyenne la production laitière après administration s'étalait de 0,6 à 1,7 L par jour. La valeur de Fisher est élevée pour le facteur animal $(F=$ 52 ) et est hautement significative $(P<0,001)$. II existe donc une forte emprise de l'animal sur la production laitière après administration de la recette. L'interaction recette $x$ animal est également significative $(P<0,05)$ pour la production laitière après administration de la recette aux vaches. Le gain laitier obtenu est hautement influencé par le type de recette $(P<0,001)$. Avec les recettes $\mathrm{R} 6$ et $\mathrm{R} 7$ à base respectivement de Curcubita maxima et de Bobgunnia madagascariensis, la production journalière maximale obtenue en milieu réel excédait $2 \mathrm{~L}$ chez certaines vaches et peut toutefois atteindre 2,5 $\mathrm{L}$ avec la recette $\mathrm{R} 7$ à base de Bobgunnia madagascariensis. Le gain laitier était aussi de 0,6 et 1,1 respectivement pour les deux recettes sus citées. 
Agani et al., J. Appl. Biosci. 2021 Préparations galactogènes utilisées par les agroéleveurs au Bénin : espèces végétales, proportions d'organes impliqués et production laitière chez les vaches Borgou

Tableau 3 : Effet des recettes sur la production laitière des vaches $(\mathrm{ml})$

\begin{tabular}{|c|c|c|c|c|}
\hline $\begin{array}{l}\text { Espèces impliquées dans la } \\
\text { préparation galactogène }\end{array}$ & Animal & $\begin{array}{l}\text { P lait Avant } \\
\text { admission }\end{array}$ & $\begin{array}{l}P \text { lait Après } \\
\text { admission }\end{array}$ & Gain laitier \\
\hline \multirow[t]{3}{*}{$R 1$} & V1 & $873,3 a$ & $1392,1 \mathrm{a}$ & $519 \mathrm{bc}$ \\
\hline & Témoins & $684,11 a$ & $688,0 \mathrm{~b}$ & \\
\hline & Moyenne & $525,5 \mathrm{BC}$ & $863,5 \mathrm{BC}$ & $338 \mathrm{BA}$ \\
\hline \multirow[t]{3}{*}{ R2 } & V1 & $662,7 a$ & $950 a$ & $287 a b$ \\
\hline & Témoins & $775 a$ & $740 \mathrm{~b}$ & \\
\hline & Moyenne & $819,2 B C$ & $1386,3 \mathrm{BA}$ & $566,8 \mathrm{~A}$ \\
\hline \multirow[t]{3}{*}{ R3 } & V1 & $1200 a$ & $1688,9 a$ & 489 bc \\
\hline & Témoins & $691 b$ & $647,5 b$ & \\
\hline & Moyenne & $996,4 \mathrm{BA}$ & $1272,3 \mathrm{BA}$ & $275,9 \mathrm{BC}$ \\
\hline \multirow[t]{3}{*}{ R4 } & V1 & $1146,7 \mathrm{a}$ & $1576 a$ & $429 \mathrm{bc}$ \\
\hline & $\mathrm{T}$ & $697,5 b$ & $781,67 a$ & \\
\hline & Moy & 967BA & $1258,7 \mathrm{BA}$ & $291,7 \mathrm{BA}$ \\
\hline \multirow[t]{3}{*}{ R5 } & V1 & 803,3 a & $1265 a$ & 462 bc \\
\hline & Témoins & $590 \mathrm{~b}$ & $635 b$ & \\
\hline & Moyenne & $718,0 \mathrm{BC}$ & $1013,0 \mathrm{BC}$ & $295 \mathrm{BA}$ \\
\hline \multirow[t]{3}{*}{$R 6$} & V1 & $1103,3 \mathrm{a}$ & $2200 \mathrm{a}$ & $1067 a$ \\
\hline & Témoins & $910 \mathrm{a}$ & $895 b$ & \\
\hline & Moyenne & $1026,0 \mathrm{BA}$ & $1678 \mathrm{~A}$ & $652 \mathrm{~A}$ \\
\hline \multirow[t]{3}{*}{ R7 } & V1 & $1810,5 \mathrm{a}$ & $2467 a$ & $657 a b c$ \\
\hline & Témoins & $850 a$ & $950 \mathrm{~b}$ & \\
\hline & Moyenne & $1330 \mathrm{~A}$ & $1708,8 \mathrm{~A}$ & $378,8 \mathrm{BA}$ \\
\hline \multirow[t]{3}{*}{ R8 } & V1 & $720 \mathrm{a}$ & $823,8 \mathrm{a}$ & $430 \mathrm{c}$ \\
\hline & Témoins & $776 a$ & $719 a$ & \\
\hline & Moyenne & $742,4 \mathrm{BC}$ & $781,9 \mathrm{BC}$ & $39,5 \mathrm{C}$ \\
\hline \multirow[t]{4}{*}{$R 9$} & V1 & $366,7 a$ & $372,5 \mathrm{a}$ & $5,8 \mathrm{c}$ \\
\hline & Témoins & $385 a$ & $646,3 b$ & \\
\hline & Moyenne & $378,9 \mathrm{C}$ & $555 \mathrm{C}$ & $176,6 \mathrm{BC}$ \\
\hline & V1 & $1146,7 a$ & $1576,7 \mathrm{a}$ & $430 \mathrm{c}$ \\
\hline \multirow[t]{2}{*}{ R10 } & Témoins & $661,25 a$ & $614,0 \mathrm{~b}$ & \\
\hline & $\begin{array}{l}\text { Moy } \\
\text { ddl }\end{array}$ & $662,1 \mathrm{BC}$ & $815,6 \mathrm{BC}$ & $153,5 \mathrm{BC}$ \\
\hline Recettes F & 9 & 4,58 & 5,33 & 7,1170 \\
\hline$P$ & & 0,0007 & 0,0002 & 0,000 \\
\hline Animal $\mathrm{F}$ & 2 & 9,22 & 52,40 & 14,68 \\
\hline$P$ & & 0,0049 & 0,0001 & 0,0313 \\
\hline Recettes*Animal F & 18 & 7,54 & 2,32 & 35,87 \\
\hline$P$ & & 0,0180 & 0,0409 & 0,0093 \\
\hline
\end{tabular}

Production maximale de lait ; $P$ : Production. Les moyennes suivies des mêmes lettres alphabétiques ne sont pas significativement différentes $(P>0,05)$. 


\section{DISCUSSION}

Espèces végétales, organes utilisés et mode de préparation des recettes galactogènes : Cette étude diagnostique sur les préparations galactogènes utilisées en milieu réel par les agroéleveurs traditionnels révèle que trois principales espèces végétales (Bobgunnia madagascariensis, Euphorbia balsamifera, Curcubita maxima) sont fréquemment impliquées dans la constitution des recettes. C'est la preuve que la flore béninoise dispose des espèces végétales successibles d'améliorer la production en lait des vaches locales élevées en milieu réel. Ces trois espèces végétales ont été précédemment citées dans la liste des plantes inventoriées par (Dassou et al., 2014; Salifou et al., 2017) dans le cadre de leur étude sur les espèces utilisées en ethno vétérinaire et les préparations galactogènes utilisées par les éleveurs pour améliorer la production du lait. Les éleveurs traditionnels sont des analphabètes mais incarnent un mode de vie traditionnel pertinent pour la conservation et l'utilisation de la biodiversité durable (Salgotra et al., 2018). Ces pratiques endogènes normalement transmis de génération en génération de bouche à oreille dans les histoires, les poèmes des proverbes et des chansons sont peu fiables mais leur procurent plusieurs avantages par rapport aux produits synthétiques qui sont chers et souvent indisponibles dans leur zone (Bullitta et al., 2018; Hussain et al., 2018). Du coût, ces autochtones pour améliorer la production de lait, utilisent une ou plusieurs combinaisons d'espèces végétales dans la préparation des recettes galactogènes. L'étude a permis de constater que des essences végétales impliquées dans les préparations galactogènes sont de la famille des légumineuses, des apocyanacés et des euphorbiacés qui sont pour la plupart des plantes à sève. Cette caractéristique de la plante, est le premier critère pour les agros éleveurs à s'assurer que la préparation pourrait être susceptible d'augmenter la production du lait chez la vache. Selon (INRA, 2014), les plantes à sève sont des plantes ayant la capacité de transporter des substances dissoutes capitales pour la production du lait chez les ruminants. II s'agit : des sels minéraux (sulfates, potassium, phosphates magnésium), des métabolites organiques (saccharose et acides aminées) et des phytohormones. Généralement, des plantes ou des parties de plantes fraîchement récoltées sont utilisées dans les préparations. Comme le souligne si bien certains auteurs (Dixit et Vakshasya, 2019; Gabalebatse et al., 2013; Lenka et al., 2018), les parties les plus couramment utilisées étaient les graines, les racines, les feuilles jeunes, et les tiges. L'utilisation de ces parties s'est expliquée par le fait que les substances actives sont bien concentrées à ce stade. Cependant, l'eau était le seul solvant utilisé pour la préparation des recettes qui étaient essentiellement faite soit par décoction ou par macération. L'adoption de ces deux modes de préparation s'explique par le fait qu'ils étaient les meilleures pour extraire le maximum de principe actif sans qu'il ne soit dénaturé ni créer des cas de pathologies chez le couple veau-vache. C'est le cas de Saba comorensis, Calotropis procera et Vitellaria paradoxa. Ces trois espèces doivent nécessairement passer à une température d'ébullition avant usage afin d'éviter que les animaux fassent la diarrhée. Ces résultats confirment ceux déjà signalés par plusieurs auteurs sur le même sujet (Atakpama et al., 2014; Bwassiwe et al., 2014; Kande et al., 2018; Mpondo et al., 2017). Tout comme dans les travaux réalisés par (Salifou et al., 2017), il a été observé une similitude dans l'utilisation des plantes, les parties de plante, la voix d'administration, le mode d'administration et le mode de préparation à travers les barrières géographiques ce qui indique une validité et une efficacité des préparations. Comme on pourrait le constater pour la plupart des préparations, les espèces végétales à graines y sont toujours ajoutées. Pour les agroéleveurs, cet ajout des graines aux recettes a une signification magicoreligieuse. Selon leur croyance, ils pensent que le nombre de graines de Vigna unguiculata ou d'Arachis hypogea ajouté aux recettes ne peut se quantifier aisément, ainsi sera également la production laitière de la vache jusqu'au tarissement. En outre, selon les croyances des peulh agro-éleveur, toutes espèces végétales dotées de sève blanche sont aussi susceptibles d'être une plante amélioratrice de la production laitière chez les vaches. L'étude diagnostique a par ailleurs révélée que la dose et la durée du traitement diffèrent d'une recette à une autre. Cela peut être dû aux différences liées à la zone agro écologique et le mode de transmission des savoirs endogènes comme l'on si bien souligné d'autres auteurs (Ahmad et al., 2015; Disler et al., 2014; Mishra, 2015; Shah et al., 2012).

Effet des préparations galactogènes sur la production laitière des vaches de race Borgou : Au regard des résultats de l'étude diagnostique, il est indiscutablement vrai que les préparations galactogènes améliorent la production de lait chez les vaches locales. La quantité de lait obtenue est fonction de la préparation galactogène. II existe donc une forte emprise de l'animal sur la production laitière après administration de la 
recette. Dans les formulations de certaines préparations galactogènes, les organes tels que les racines sont utilisées. Mais, la récolte des racines de plantes pour ces préparations pose des problèmes d'écologie (Betti, 2002; Walker et al., 1994), car les plantes sont détruites par la récolte de ces organes (Dro et al., 2013). Par ailleurs, les quantités des différents organes dans la préparation galactogène varient très fortement en fonction de la recette, de l'éleveur et de la zone agroécologique. Le fait que les graines de légumineuses soient toujours associées aux préparations galactogènes trouve toute sa justification dans le domaine de la science exacte. En effet, les légumineuses à graines sont très connues comme des plantes ayant des valeurs nutritives importantes qui participent à la reconstitution et à la croissance des cellules animales par leur profil en calcium, en phosphore, en magnésium et en vitamine $A$ et $C$ (Agugo et al., 2013). Leurs teneurs en fer sont égales à celle du lait (Sayeed et al., 2017). La richesse en Fer et en vitamine B12 de ces légumineuses fait qu'une fois après parturition ajoutée aux recettes renforcent l'organisme par le mécanisme du cycle de Krebs qui améliorent à son tour la performance laitière des vaches (Evans et al., 2010; Oppong Bekoe et al., 2020). II a été également prouvé par (Sayeed et al., 2017), que Vigna unguiculata

\section{CONCLUSION}

L'étude révèle que les éleveurs utilisent effectivement des préparations galactogènes pour améliorer la production du lait chez les vaches locales. C'est la preuve que la flore béninoise dispose des espèces végétales amélioratrices de la production. Parmi ces espèces végétales il en existe qui sont toujours fréquentes dans les préparations galactogènes. II s'agit de Bobgunnia madagascariensis, Curcubita maxima, Euphorbia basalmifera. Quelle que soit la recette les éleveurs associent les graines de Vigna unguiculata ou de Arachis hypogea. Avec la recette à base de Bobgunnia madagascariensis la production maximale de lait est de 2,5 litres contre 1,5 en élevage semi-intensif.

\section{REMERCIEMENT}

Les auteurs remercient le Programme Fonds Compétitifs de Recherche de l'Université d'Abomey-Calavi pour son appui financier et l'équipe du Projet PROLAIT pour son possède des propriétés pharmacologiques diversifiées dont l'effet galactagogue. Tout porte à croire que sans certains organes de plantes telles que Bobgunnia madagascariensis, Curcubita maxima, Euphorbia basalmifera et Vigna unguiculata, l'efficacité de la préparation galactogène n'est pas garantie. La littérature rapporte que les plantes galactogènes impliquées dans les préparations sont riches en constituants chimiques tels que les flavonoïdes, les tanins, les polyphénols, les alcaloïdes et les triterpènes ayant des activités antimicrobiennes, antivirales, analgésiques, antyrétiques et anti-inflammatoire anti-parasitaires agissent en synergie pour améliorer la santé des animaux ainsi les capacités reproductrices des vaches. Par ailleurs, Sorghum bicolor (L), Arachis hypogea, Vigna unguiculata, et Calotropis procera (Ait.) ont fait objet de certaines études sur leur effet à induire la lactation chez les mammifères (Koko et al., 2011; Sayeed et al., 2017). II est aussi primordial de montrer qu'à part leur propriété phytochimique Arachis hypogea, et Vigna unguiculata ont un meilleur profil en minéraux (rapport $\mathrm{Ca} / \mathrm{P}$ ) ; magnésium ; vitamine $\mathrm{A}$; $\mathrm{B} 12$ et $\mathrm{C}$ qui sont essentiels à la monté lactée donc à l'éjection du lait des canaux galactophores (Agugo et al., 2013; Sayeed et al., 2017).

Le gain laitier journalier suite à l'administration des recettes s'étale entre 0,6 et 1,1 litre. II ne reste maintenant qu'à identifier pour les recettes la forme galénique la mieux adaptée aux conditions socioéconomiques des éleveurs traditionnels. Par ailleurs, les plantes impliquées dans la constitution des préparations retrouvées dans la nature, courent au fil du temps des menaces dues aux expansions agricoles. En outre, les formulations de certaines préparations galactogènes nécessitent le prélèvement de certains organes tels que les racines. C'est un véritable problème écologie qu'il faudra résoudre car les plantes sont détruites par la récolte de ces organes.

appui technique. Les auteurs remercient également les éleveurs des communes de Gogounou, Kalalé et Djidja pour leur soutien technique et professionnel.

\section{CONFLIT D'INTÉRÊT}

Les auteurs déclarent qu'il n'y a pas de conflit d'intérêts par rapport à cet article. 


\section{RÉFÉRENCES}

Agugo, U.A., Okere, T.O., Anya, K.M., 2013. Investigating the Nutrient Composition and Anti-nutritional Factors of Akidi (Vigna unguiculata). IOSR J. Environmental Sci. Toxicol. Food Technol. 5, 32-35.

Ahmad, K., Ahmad, M., Weckerle, C., 2015. Ethnoveterinary medicinal plant knowledge and practice among the tribal communities of Thakht-e-Sulaiman hills, west Pakistan. J. Ethnopharmacol. 170, 275-283.

Akouedegni, C.G., Tossa, I.G., Daga, F.D., Koudandé, D.O., 2012. Synthèse des connaissances sur les plantes galactogènes et leurs usages en République du Bénin. Line 12.

Akouègninou, A., Van der Burg, W.J., van der Maesen, L.J.G., 2006. Flore analytique du Bénin. Cotonou \& Wageningen. Edition Backhuys Publishers.

Atakpama, W., Kponor, E.G.E., Kanda, M., Dourma, M., M'tékounm, N., Batawila, K., Akpagana, K., 2014. Moringa oleifera Lamarck (Moringaceae): une ressource phytogénétique à usage multiple. Sci. Vie Terre Agron. 2.

Betti, J.L., 2002. Medicinal plants sold in Yaoundé markets, Cameroon.

Bullitta, S., Re, G.A., Manunta, M.D.I., Piluzza, G., 2018. Traditional knowledge about plant, animal, and mineral-based remedies to treat cattle, pigs, horses, and other domestic animals in the Mediterranean island of Sardinia. J. Ethnobiol. Ethnomedicine 14, 50.

Bwassiwe, H., Metowogo, K., Aklesso, P., Mouzou, R., Tossou, R., Ahounou, J., Eklu-Gadegbekou, K., Dansou, P., Aklikokou, K., 2014. Enquête ethnobotanique sur les plantes utilisées dans le traitement traditionnel des contusions musculaires au Togo. Rev. Ivoirienne Sci. Technol. 24, 112-130.

Dagnelie, P., 1973. Théorie et méthodes statistiques. Applications agronomiques. 1, La statistique descriptive et les fondements de l'inférence statistique. J. Duculot.

Dassou, H.G., Ogni, C.A., Yédomonhan, H., Adomou, A.C., Tossou, M., Dougnon, J.T., Akoègninou, A., 2014. Diversité, usages vétérinaires et vulnérabilité des plantes médicinales au NordBénin. Int. J. Biol. Chem. Sci. 8, 189-210.

Disler, M., Ivemeyer, S., Hamburger, M., Vogl, C.R., Tesic, A., Klarer, F., Meier, B., Walkenhorst, M., 2014. Ethnoveterinary herbal remedies used by farmers in four north-eastern Swiss cantons (St. Gallen, Thurgau, Appenzell Innerrhoden and Appenzell Ausserrhoden). J. Ethnobiol. Ethnomedicine 10, 32.

Dixit, G., Vakshasya, S., 2019. Ethnoveterinary Medicinal Plants Used by Ethnic and Rural People of Indo-Nepal Sub Himalayan International Border Region of Pilibhit Tiger Reserve (PTR), Uttar Pradesh, India. Plantae Sci. 2, 15-18.

Dossou, M.E., Houessou, G.L., Lougbégnon, O.T., Tenté, A.H.B., Codjia, J.T.C., 2012. Etude ethnobotanique des ressources forestières ligneuses de la forêt marécageuse d'Agonvè et terroirs connexes au Bénin. Tropicultura 30.

Dro, B., Soro, D., Koné, M.W., Bakayoko, A., Kamanzi, K., 2013. Évaluation de l'abondance de plantes médicinales utilisées en médecine traditionnelle dans le Nord de la Côte d'Ivoire. J. Anim. Plant Sci. 17, 2631-2646.

Evans, F.J., McMurtry, D.R., Bulled, C.R., 2010. Metrology apparatus.

FAO, 2013. CountrySTAT - Benin [WWW Document]. URL

http://countrystat.org/home.aspx?c=BEN\&ta=0 53CPD035\&tr=7 (accessed 10.6.20).

Gabalebatse, M., Ngwenya, B.N., Teketay, D., Kolawole, O.D., 2013. Ethno-veterinary practices amongst livestock farmers in Ngamiland District, Botswana. Afr. J. Tradit. Complement. Altern. Med. 10, 490-502.

Hussain, W., Badshah, L., Ullah, M., Ali, M., Ali, A., Hussain, F., 2018. Quantitative study of medicinal plants used by the communities residing in Koh-e-Safaid Range, northern Pakistani-Afghan borders. J. Ethnobiol. Ethnomedicine 14, 1-18.

INRA, 2014. Inra Productions Animales - 2014 - Volume 27 [WWW Document]. URL https://www6.inrae.fr/productionsanimales/2014-Volume-27 (accessed 10.8.20).

Kande, B., Yao, K., Allah-Kouadio, E., Kone, M.W., 2018. Enquête sur l'utilisation et l'effet des médicaments à base de plantes chez les patients hépatiques hospitalisés au Service de médecine et d'hépatogastroentérologie du Centre Hospitalier Universitaire (CHU) de Cocody en Côte d'Ivoire. J. Appl. Biosci. 130, 13220-13231. 
Koko, I.K.D., Djego, J., Gbenou, J., Hounzangbé-Adoté, S.M., Sinsin, B., 2011. Etude phytochimique des principales plantes galactogènes et emménagogues utilisées dans les terroirs riverains de la Zone cynégétique de la Pendjari. Int. J. Biol. Chem. Sci. 5.

Lenka, K.C., Pradhan, N., Padhan, B., 2018. Ethnoveterinary Medicines: AP otential Alternative to Animal Health Care for the Tribal Communities of Koraput, Odisha. Int. J. Pharmacol. Phytochem. Ethnomedicine 26.

Mama Sambo, A., 2013. Etude relative à la formulation du programme d'actions détaillé du développement de la filière lait en zone UEMOA. Cirad Montp. Fr.

Mishra, D.P., 2015. An ethnoveterinary survey of medicinal preparations used to treat painful delivery and retention of placenta in domestic cattle in Polasara block, Ganjam district, Odisha, India. J Livest Sci 5.

Mpondo, E.M., Ngene, J.P., Som, L.M., Loe, G.E., Boumsong, P.C.N., Yinyang, J., Dibong, S.D., 2017. Connaissances et usages traditionnels des plantes médicinales du département $d u$ haut Nyong. J. Appl. Biosci. 113, 11229-11245.

Oppong Bekoe, E., Agyare, C., Boakye, Y.D., Baiden, B.M., Asase, A., Sarkodie, J., Nettey, H., Adu, F., Otu, P.B., Agyarkwa, B., Amoateng, P., Asiedu-Gyekye, I., Nyarko, A., 2020. Ethnomedicinal survey and mutagenic studies of plants used in Accra metropolis, Ghana. J. Ethnopharmacol. 248, 112309. https://doi.org/10.1016/j.jep.2019.112309

Salgotra, R.K., Zargar, S.M., Sharma, M., Sood, M., 2018. Traditional Knowledge: A Therapeutic Potential in the Scenario of Climate Change for Sustainable Development. Development 61, 140-148.

Salifou, C.F.A., Kassa, K.S., Ahounou, S.G., Moussa, H., Dotché, I.O., Agbozo, J.M., Issifou, M.T., Youssao, I.A.K., 2017. Plantes lactogènes des bovins et leurs modes de préparation dans les élevages traditionnels au Bénin. Livest. Res. Rural Dev. 29, 29.

Sayeed, M.A., Bracci, M., Lazzarini, R., Tomasetti, M., Amati, M., Lucarini, G., Di Primio, R., Santarelli, L., 2017. Use of potential dietary phytochemicals to target miRNA: promising option for breast cancer prevention and treatment? J. Funct. Foods 28, 177-193.
Shah, G.M., Ahmad, M., Arshad, M., Khan, M.A., Zafar, M., Sultana, S., 2012. Ethno-phyto-veterinary medicines in northern Pakistan. J. Anim. Plant Sci. 22, 791-797.

Walker, M.D., Walker, D.A., Auerbach, N.A., 1994. Plant communities of a tussock tundra landscape in the Brooks Range Foothills, Alaska. J. Veg. Sci. 5, 843-866. 